\title{
GEOMETRIC AND HIERARCHICAL FDI FOR THE IFATIS TWO-TANK PILOT PLANT
}

\author{
Raffaella Mattone and Alessandro De Luca
}

\author{
Dipartimento di Informatica e Sistemistica \\ Università di Roma "La Sapienza”, Italy \\ E-mail:\{mattone,deluca\}@dis.uniroma1.it
}

\begin{abstract}
In the control of complex dynamic plants, fault detection and isolation (FDI) is a prerequisite for a fault tolerant control architecture. In order to increase the reliability and robustness of the fault diagnosis, it is fundamental that the FDI system is given a hierarchical structure, reflecting, as far as possible, the natural decomposition of the plant into physical and/or logical subsystems. Through the use of the IFATIS two-tank benchmark, we illustrate the design and implementation of a hierarchical system for the detection and isolation of non-concurrent faults of sensors and actuators, based on the processing of suitably selected residuals, designed with geometric techniques. The FDI performance is demonstrated by realistic simulations performed on the identified model of the real system, including actual levels of input and measurement noise. Copyright (C) 2005 IFAC
\end{abstract}

Keywords: Fault detection, fault isolation, hierarchical systems, nonlinear systems.

\section{INTRODUCTION}

In the control of complex dynamic plants, the problem of automatically detecting the occurrence of a faulty behavior in one or more of the hardware components is crucial for a reliable continuous operation. In fact, fault detection and isolation (FDI) is a prerequisite for a fault tolerant control architecture: when the system supervisor has recognized the type and location of a fault, it can activate a reconfiguration of the sensing, actuating, communication, and control devices so as to minimize performance degradation or even recover full operation.

An FDI system is typically a dynamic system where each output signal (residual) is excited in response to the occurrence of a different fault, as a result of the processing of nominal commanded inputs and measured outputs of the monitored plant. The set of available sensors and actuators may be fixed, or be a sub-product of the control and FDI design, whenever the integrated design of the overall system is allowed. A natural requirement for the FDI system is that it is able to diagnose the failure of any hardware device used to control the system and/or detect and isolate a fault, at least under the assumption of nonconcurrency of faults. Whenever a model of the nominal (faultless) plant is available, the residual generation naturally relies on the knowledge of the static and/or dynamic equations governing the system. A relevant example in the class of modelbased FDI methods is constituted by the geometric techniques introduced in (De Persis and Isidori, 2001) and recently developed and extended in (Mattone and De Luca, 2003 and 2004), that are applicable to a wide class of nonlinear systems affine in the (control and fault) inputs.

The reliability of the designed FDI system (and thus, of the whole fault-tolerant control system) depends on the accuracy of the available model and on the quality of the used hardware equipment (sensors and actuators). In order to increase the 
overall reliability and robustness of fault diagnosis (e.g., by limiting the propagation effects of false/missed FDI alerts), but also to optimize the distribution of the computational load and improve the modularity and reusability of the code, it is fundamental that the FDI module is given a hierarchical structure, reflecting, as far as possible, the natural decomposition of the control system into physical or logical subsystems (Bonivento et al., 2004). Most FDI techniques, including geometric methods, do not allow the automatic inclusion of such "structural" requirements in the design, so that the obtained residual generators must be reorganized "by hand", in order to fit a particular system architecture. On the other hand, geometric techniques provide analysis tools that may support the selection of suitable subsets of residuals within the set of all independent diagnostic signals that can be generated for the considered system. Thus, the desired architecture can be established for the final FDI system, before the actual design of residuals.

Through the use of a simple case study (a twotank pilot plant used as benchmark in the IFATIS project, see Sect. 2), we illustrate in this paper the design and implementation of a geometric, hierarchical system for the detection and isolation of non-concurrent faults of sensors and actuators (Sects. 3-4). In particular, we show how the needed set of measurements follows from the requirements on the control and FDI systems. The performance of the designed FDI system is demonstrated in Sect. 5 by realistic simulations for an identified model of the real plant, including the actual levels of input and measurement noise.

\section{THE IFATIS TWO-TANK PILOT PLANT}

We consider hereafter the system schematized in Fig. 1, corresponding to a real pilot plant used as benchmark in the EU Project IFATIS. It is constituted by two tanks, with fluid levels $L_{1}$ and $L_{2}$, connected by a controlled interconnection valve. The control inputs are the flows $Q_{1}$ and $Q_{2}$, fed by hydraulic pumps driven by tensions $V_{1}$ and $V_{2}$, and the (inverse of the) throttling $R_{12}$ of the interconnection valve, driven by the input tension $V_{12}$. The controlled outputs are the sum and ratio of the tank output flows $Q_{F 1}$ and $Q_{F 2}$, directly related to $L_{1}$ and $L_{2}$ through the nonlinear characteristics of the output valves. The evolution of the state variables $L_{1}$ and $L_{2}$ is modelled by

$$
\begin{gathered}
S_{1} \dot{L}_{1}=Q_{1}-Q_{12}-Q_{F 1}, \\
S_{2} \dot{L}_{2}=Q_{2}+Q_{12}-Q_{F 2},
\end{gathered}
$$

where the known nonlinear mappings

$$
Q_{1}=\pi_{1}\left(V_{1}\right), \quad Q_{2}=\pi_{2}\left(V_{2}\right),
$$

$$
Q_{F 1}=\varphi_{1}\left(L_{1}\right), \quad Q_{F 2}=\varphi_{2}\left(L_{2}\right)
$$

and

$$
Q_{12}=\frac{1}{R_{12}\left(V_{12}\right)} \cdot \varphi_{12}\left(\left|L_{1}-L_{2}\right|\right) \cdot \operatorname{sign}\left(L_{1}-L_{2}\right),
$$

describe the identified characteristics of the pumps and valves as a function of the corresponding input voltages and fluid levels, and $S_{1}, S_{2}$ are the sections of Tank 1 and Tank 2, respectively (see Hamelin et al. (2004) for details). For this system, measures of the two tank levels $L_{1}$ and $L_{2}$, and of the flows $Q_{F 1}, Q_{F 2}$ and $Q_{12}$ are available. The effects of all disturbances and model mismatches have been modelled as additive noise on the measured outputs, that resulted to be described by zero mean normal distributions with standard deviations $s_{L 1}=s_{L 2}=0.015, s_{Q F 1}=s_{Q F 2}=0.034$, and $s_{Q 12}=0.037$.

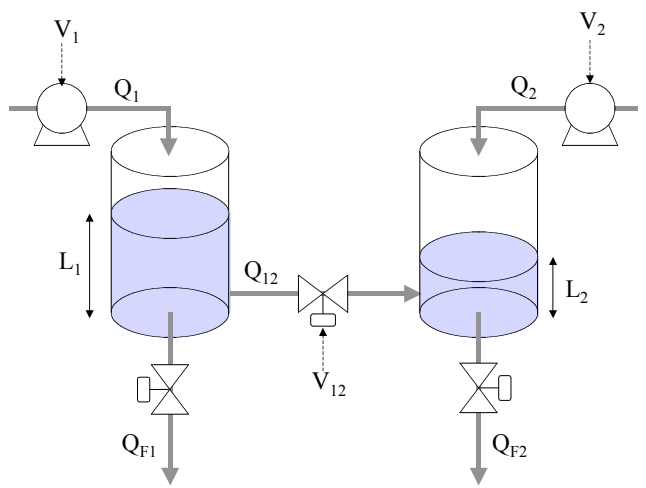

Fig. 1. The 2-tank pilot plant.

\section{DESIGN OF THE FDI SYSTEM}

The fault diagnosis system for the considered plant is based on the following requirements and realistic assumptions:

1. The FDI system must be able to operate in any dynamic situation, i.e., at steady state as well as during the control transient.

2. No special time profile for the fault quantities is assumed.

3. Any hardware device used to control the system and/or detect and isolate a fault, may fail itself.

4. The probability of concurrent failure of different hardware devices is negligible.

5. The structure of the FDI system should reflect as close as possible the natural decomposition of the controlled plant into the local subsystems constituted by the two tanks, each characterized by the local quantities $Q_{i}, L_{i}$, 
$Q_{F i}, i=1,2$, and having in common just the input $R_{12}$ and the measurement $Q_{12}$.

As a first step in the design of the FDI system, we just consider the hardware devices that are used for controlling the system, i.e., the two pumps providing $Q_{1}$ and $Q_{2}$, the interconnection valve with throttling $R_{12}$, and the two sensors for levels $L_{1}$ and $L_{2}$. According to requirement 3, all considered devices might be affected by faults. We define then the following fault quantities:

$$
\begin{gathered}
f_{Q 1}=Q_{1 a}-Q_{1 c}, \quad f_{Q 2}=Q_{2 a}-Q_{2 c}, \\
f_{R 12}=\frac{1}{R_{12 a}}-\frac{1}{R_{12 c}}, \\
f_{L 1}=L_{1 a}-L_{1 m}, \quad f_{L 2}=L_{2 a}-L_{2 m},
\end{gathered}
$$

where subscripts ' $a$ ', ' $c$ ' and ' $m$ ' stay for actual, commanded, and measured, respectively.

From the geometric approach of Mattone and De Luca (2003) it follows that, with the chosen set of measures, a maximum number of three independent residual generators can be designed for the considered system affected by the faults in (6). The resulting residual matrix ${ }^{1}$ is given in Table 1. A possible set of such residual generators is reported below ${ }^{2}$, where only available values (i.e., commanded or measured) are used (subscripts are omitted ):

$$
\begin{aligned}
\dot{\xi}_{1} & =\frac{1}{S} \pi_{1}\left(V_{1}\right)-\frac{1}{S} \frac{\varphi_{12}\left(\left|L_{1}-L_{2}\right|\right)}{R_{12}\left(V_{12}\right)} \cdot \operatorname{sign}\left(L_{1}-L_{2}\right) \\
& -\frac{1}{S} \varphi_{1}\left(L_{1}\right)+K_{1}\left(L_{1}-\xi_{1}\right) \\
r_{1} & =L_{1}-\xi_{1}, \\
\dot{\xi}_{2} & =\frac{1}{S} \pi_{2}\left(V_{2}\right)+\frac{1}{S} \frac{\varphi_{12}\left(\left|L_{1}-L_{2}\right|\right)}{R_{12}\left(V_{12}\right)} \cdot \operatorname{sign}\left(L_{1}-L_{2}\right) \\
& -\frac{1}{S} \varphi_{2}\left(L_{2}\right)+K_{2}\left(L_{2}-\xi_{2}\right) \\
r_{2}= & L_{2}-\xi_{2}, \\
\xi_{3} & =\frac{1}{S} \pi_{1}\left(V_{1}\right)+\frac{1}{S} \pi_{2}\left(V_{2}\right)-\frac{1}{S} \varphi_{1}\left(L_{1}\right) \\
& -\frac{1}{S} \varphi_{2}\left(L_{2}\right)+K_{3}\left(L_{1}+L_{2}-\xi_{3}\right) \\
r_{3} & =L_{1}+L_{2}-\xi_{3},
\end{aligned}
$$

\footnotetext{
1 This matrix, whose entry $(i, j)$ is ' 1 ' if the $i$-th fault affects the $j$-th residual, can be computed on the basis of the system and fault vector fields, before the actual design of residual generators.

${ }^{2}$ Input and output noise has not been considered in the design, but will be suitably taken into account in the logical processing of residuals.
}

with $K_{i}>0, i=1, \ldots, 3$. It can be readily verified that the residual generators in eqs. (7-9) are nonlinear observers for the dynamics of the variables $L_{1}, L_{2}$ and $L_{1}+L_{2}$, respectively, and that, in the absence of faults, the dynamics of the residuals satisfies

$$
\dot{r}_{i}=-K_{i} r_{i}, \quad i=1, \ldots, 3 .
$$

By looking at the residual matrix in Table 1, one realizes that the three designed residual generators are not sufficient to isolate all potential faults affecting the system. In particular, it is not possible to discriminate between the failures $f_{L 1}$ and $f_{L 2}$ of the two level sensors, since they affect the same set of residuals (the corresponding rows of the residual matrix are equal).

Table 1. Residual matrix relating faults $f_{\underline{Q 1}}, \ldots, f_{\underline{L 2}}$ of eq. (6) to the residuals $r_{1}, \ldots, r_{3}$ of eqs. (7-9)

Residual $\begin{array}{rrr}r_{1} & r_{2} & r_{3}\end{array}$

Fault

\begin{tabular}{cccc}
\hline$f_{Q 1}$ & 1 & 0 & 1 \\
$f_{Q 2}$ & 0 & 1 & 1 \\
$f_{R 12}$ & 1 & 1 & 0 \\
$f_{L 1}$ & 1 & 1 & 1 \\
$f_{L 2}$ & 1 & 1 & 1 \\
\hline
\end{tabular}

In order to get further diagnostic signals, the available measures of flows $Q_{F 1}$ and $Q_{F 2}$ can be used. As a result, the following static residual generators can be also defined

$$
\begin{aligned}
& r_{4}=Q_{F 1}-\varphi_{1}\left(L_{1}\right), \\
& r_{5}=Q_{F 2}-\varphi_{2}\left(L_{2}\right) .
\end{aligned}
$$

Correspondingly, the two fault quantities

$$
f_{Q F 1}=Q_{F 1 a}-Q_{F 1 m}, \quad f_{Q F 2}=Q_{F 2 a}-Q_{F 2 m},
$$

must be also considered, associated to the possible failure of the introduced flow sensors. It can be readily verified that, with the introduction of the two further residuals $r_{4}$ and $r_{5}$, all rows of the resulting residual matrix (corresponding to columns 1,3,5-7 of Table 2) are different, so that all considered faults may be detected and isolated by processing the residuals $r_{1}, \ldots, r_{5}$. However, in order to gain further freedom in the optimization of the FDI system, one can exploit also the information provided by the available sensor for flow $Q_{12}$. The following further diagnostic signal is introduced 


$$
r_{6}=\left|Q_{12}\right|-\frac{\varphi_{12}\left(\left|L_{1}-L_{2}\right|\right)}{R_{12}\left(V_{12}\right)},
$$

together with the fault quantity

$$
f_{Q 12}=Q_{12 a}-Q_{12 m} \text {. }
$$

Moreover, the measure of flow $Q_{12}$ can be used in eqs. (7-8) instead of the model-based term (5), in order to get alternative definitions $r_{1}^{\prime}$ and $r_{2}$ ' of residuals $r_{1}$ and $r_{2}$. These are more convenient for the purpose of a hierarchical decomposition of the FDI system. In fact, in $r_{1}^{\prime}$ and $r_{2}^{\prime}$ no crossdependence on the local level measurements $L_{1}$ and $L_{2}$ are present. At this stage, the residual matrix is given in Table 2 .

Table 2. Residual matrix relating faults $f_{Q 1}, \ldots, f_{Q 12}$ to residuals $r_{1}, \ldots, r_{6}, r_{1}^{\prime}, r_{2}{ }_{-}^{\prime}$

Residual $\quad r_{1} \quad r_{1}^{\prime} \quad r_{2} \quad r_{2}^{\prime} \quad r_{3} \quad r_{4} \quad r_{5} \quad r_{6}$

Fault

\begin{tabular}{lllllllll}
\hline$f_{O 1}$ & 1 & 1 & 0 & 0 & 1 & 0 & 0 & 0 \\
$f_{Q 2}$ & 0 & 0 & 1 & 1 & 1 & 0 & 0 & 0 \\
$f_{R 12}$ & 1 & 0 & 1 & 0 & 0 & 0 & 0 & 1 \\
$f_{L 1}$ & 1 & 1 & 1 & 0 & 1 & 1 & 0 & 1 \\
$f_{L 2}$ & 1 & 0 & 1 & 1 & 1 & 0 & 1 & 1 \\
$f_{Q F 1}$ & 0 & 0 & 0 & 0 & 0 & 1 & 0 & 0 \\
$f_{Q F 2}$ & 0 & 0 & 0 & 0 & 0 & 0 & 1 & 0 \\
$f_{Q 12}$ & 0 & 1 & 0 & 1 & 0 & 0 & 0 & 1 \\
\hline
\end{tabular}

Clearly, not all residuals in Table 2 have to be implemented. In particular, the selected columns of the residual matrix must have all different rows (to allow fault isolation under the assumption of non-concurrency) and, possibly after reordering rows and columns, must exhibit a block-diagonal structure (in order to recover the desired modular and hierarchical architecture for the FDI system). It is readily verified that these requirements are fulfilled by the set of residuals $\left\{r_{1}{ }^{\prime}, r_{4}, r_{6}, r_{2}{ }^{\prime}, r_{5}\right\}$, as in Table 3. As a consequence of this structure, the designed FDI system has the modular and hierarchical structure shown in Fig. 2, with the eight outputs one-to-one corresponding to the faults listed in Table 3. The two local FDI modules are in charge of computing the residuals that are only affected by local quantities, respectively $r_{1}{ }^{\prime}, r_{4}$ for Local FDI 1 (tank 1) and $r_{2}$,' $r_{5}$ for Local FDI 2 (tank 2). Residual $r_{6}$ has to be computed by a centralized FDI Manager, since it requires the availability also of common quantities. The FDI modules in Fig. 2 contain also the isolation logics, which is a combinatorial mapping of each feasible set of affected and unaffected residuals into a single isolated fault. In particular, the evaluation of local residuals allow the isolation of local faults of the tank level and output-flow sensors, while the discrimination of faults of the input pumps or of the interconnection valve needs the further evaluation of residual $r_{6}$ and thus the intervention of the FDI Manager.

Table 3. Residual matrix/isolation logics relating faults $f_{Q 1}, \ldots, f_{Q F 2}$ to residuals $r_{1}{ }^{\prime}, r_{4}, r_{6}, r_{2}{ }^{\prime}, r_{5}$. The block-diagonal structure has been evidenced

Residual $\quad r_{1}^{\prime} \quad r_{4} \quad r_{6} \quad r_{2}^{\prime} \quad r_{5}$

Fault

\begin{tabular}{llllll}
\hline$f_{Q 1}$ & 1 & 0 & 0 & 0 & 0 \\
$f_{L 1}$ & 1 & 1 & 1 & 0 & 0 \\
$f_{Q F 1}$ & 0 & 1 & 0 & 0 & 0 \\
$f_{R 12}$ & 0 & 0 & 1 & 0 & 0 \\
$f_{Q 12}$ & 1 & 0 & 1 & 1 & 0 \\
$f_{Q 2}$ & 0 & 0 & 0 & 1 & 0 \\
$f_{L 2}$ & 0 & 0 & 1 & 1 & 1 \\
$f_{Q F 2}$ & 0 & 0 & 0 & 0 & 1 \\
\hline
\end{tabular}

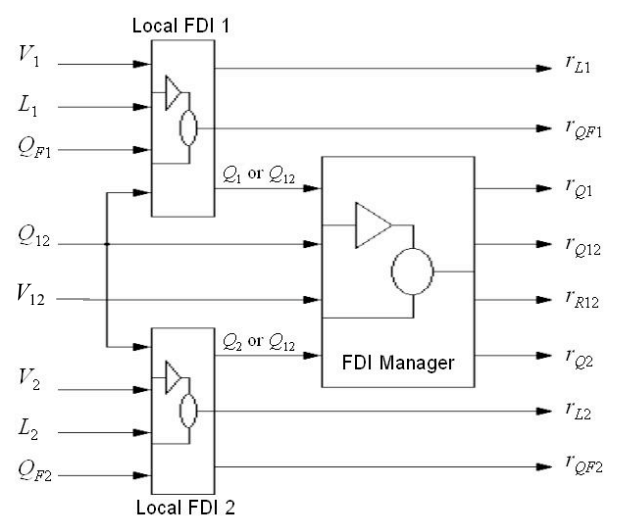

Fig. 2. Hierarchical structure of the designed FDI system.

\section{IMPLEMENTATION DETAILS}

The FDI system described in Sect. 3 has been implemented using Simulink. The used dynamic equations in the FDI system have been discretized by Euler method, with sampling time $T=1 \mathrm{~s}$. In the dynamics of $r_{1}{ }^{\prime}$ and $r_{2}{ }^{\prime}$, observation gains $K_{1}=K_{2}=0.1$ were found satisfactory. In order to deal with unmodelled input and measurement noise, the selected analogic residuals are processed by the following dynamic thresholding algorithm:

1. Each residual $r$ in the columns of Table 3 is compared with a fixed threshold, defined from the observed amplitude of the residual in the absence of faults, resulting in a boolean residual $r$ dig. The chosen thresholds were, respectively, 0.04, 0.05, 0.04, 0.08 and 0.08 . 

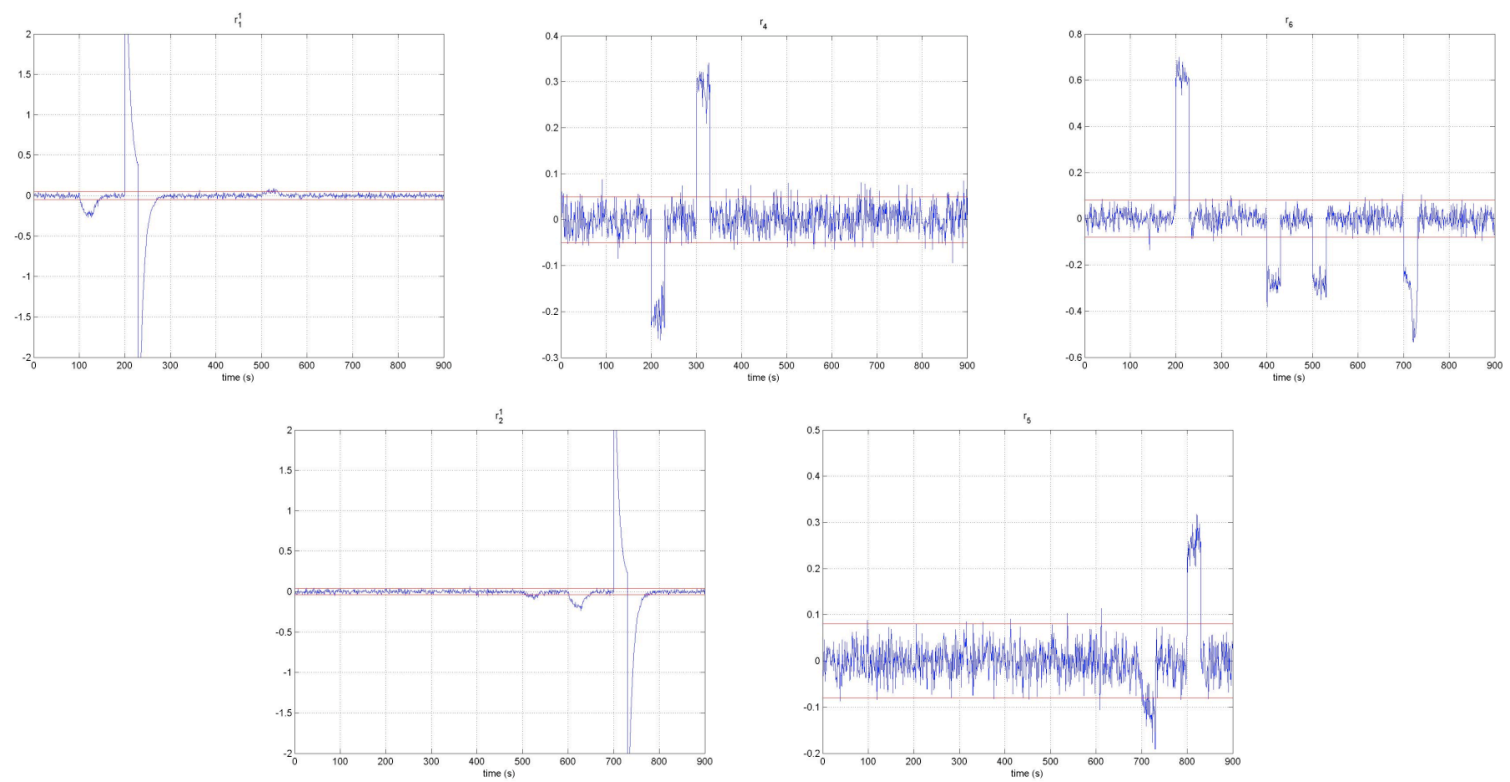

Fig. 3. Behaviour of residuals $r_{1}{ }^{\prime}, r_{4}, r_{6}, r_{2}{ }^{\prime}, r_{5}$ (from top left to bottom right). Residual thresholds are also indicated.

2. Each residual $r$ dig is filtered to eliminate spurious ' 1 ' and ' 0 ', or oscillations between these two values. This is obtained by switching the residual value only after it has been stable on the new value for a fixed number of samples (respectively, corresponding to $T_{\text {on }}=2 \mathrm{~s}$ and $T_{\text {off }}=5 \mathrm{~s}$ ). The resulting signal is $r$ filt.

3. The isolation logics is then evaluated on $r$ filt, providing the final boolean output vector $r_{-}$isol $=\left(r_{Q 1}, \ldots, r_{Q F 2}\right)$. In order to deal with the different residual dynamics and avoid incorrect isolation during transients, the evaluation of the combinatorial mapping of Table 3 is enabled only after at least one of the $r$ filt components has been 'on' for $T_{\text {detect }}=8 \mathrm{~s}$. For the same reason, $r$ isol is reset to zero only after vector $r$ filt has been 'off' for at least $T_{\text {reset }}=2 \mathrm{~s}$. Isolation is considered unsuccessful if it has been enabled, but no component of $r_{-}$isol has been set to ' 1 '.

At the cost of a small delay in the diagnosis, this algorithm adds robustness to the FDI scheme.

\section{SIMULATION RESULTS}

The implemented FDI system has been tested on the identified model of the real plant given by Hamelin et al. (2004). The system was under open-loop control, being, in particular,

$$
\begin{aligned}
& V_{1}=2.7(1+0.4 \sin 0.02 \pi t), \\
& V_{2}=1.4983(1+0.4 \sin 0.02 \pi t), \\
& V_{12}=6 .
\end{aligned}
$$

The simulated scenario was a sequence of nonconcurrent faults occurring in the same order used to list the faults in Table 3 , at times $t_{i}=100 \cdot i \mathrm{~s}$, $i=1, \ldots, 8$. In all cases, the fault consists of a constant bias on the actuator input voltage or on the sensor output voltage, respectively, with a fault duration of $30 \mathrm{~s}$. In particular, with obvious meaning of symbols, it has been set

bias_V1 $=0.5[\mathrm{~V}] \quad(10 \%$ of available range $)$ bias L1 $=3.16$ [V] $(40 \%$ of available range $)$ bias_QF1 $=0.2922[\mathrm{~V}](40 \%$ of available range $)$ bias_V12 $=-6 \quad[\mathrm{~V}] \quad(100 \%$ of available range $)$ bias_Q12 $=0.28 \quad[\mathrm{~V}] \quad(15 \%$ of available range $)$ bias_V2 $=0.5[\mathrm{~V}] \quad(10 \%$ of available range $)$ bias_L2 $=3.02[\mathrm{~V}] \quad(40 \%$ of available range $)$ bias_QF2 $=0.249[\mathrm{~V}] \quad(30 \%$ of available range $)$ 

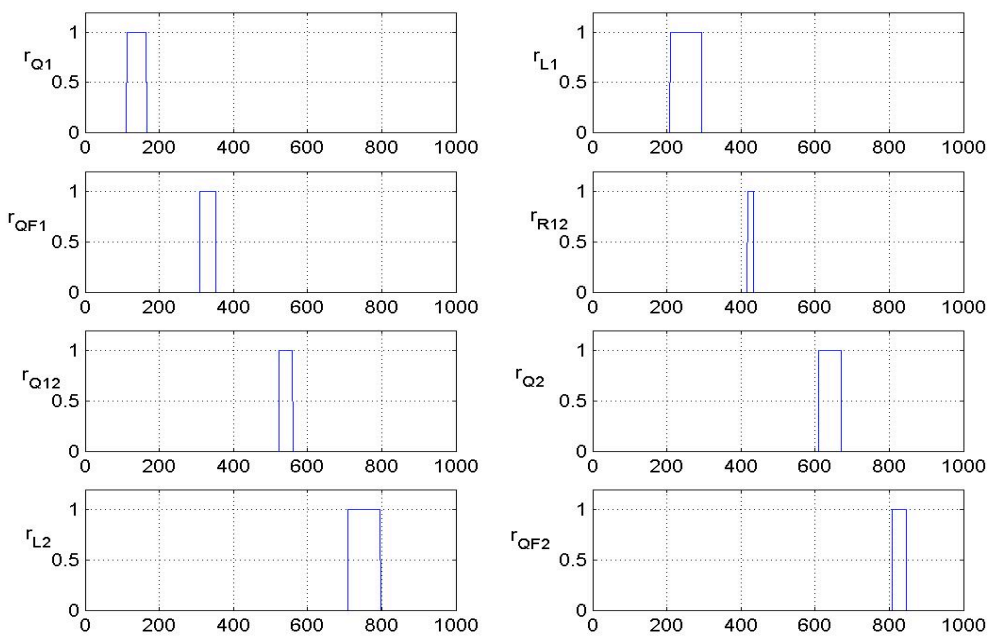

Fig. 4. Behaviour of residuals $r_{Q 1}, \ldots, r_{Q F 2}$ (from top left to bottom right).

The differences in the chosen fault severities depend on the different sensitivity of the FDI system to each fault. In particular, the biases were empirically chosen to be approximately twice the minimum values that can be detected and isolated with acceptable reliability. The only critical fault is that of the interconnection valve. In fact, the sensitivity to this actuator fault basically depends on the levels $L_{1}$ and $L_{2}$. We can only say that, with the given FDI design, the minimum detectable bias on the flow $Q_{12}$ through the valve is $0.2310^{-4}$ $\left[\mathrm{m}^{3} / \mathrm{s}\right]$ (or $0.14[\mathrm{~V}]$, expressed in the units of the flow sensor).

The behaviour of the relevant diagnostic signals during the described fault scenario is shown in Figs. 3-4. In particular, it can be observed in Fig. 3 that a set of multiple residuals is excited by the occurrence of each fault, in agreement with Table 3. In Fig. 4, the final boolean outputs of the FDI system show a correct fault isolation. The delays in the individuation of the fault intervals are due to the dynamic thresholding mechanism.

\section{CONCLUSIONS}

Through the use of a nonlinear case study (the IFATIS two-tank pilot plant), we have illustrated the design and implementation of a modular and hierarchical FDI system for the detection and isolation of actuator and sensor faults. Starting from the natural requirements and realistic assumptions for the diagnostic system, we have motivated the use of further sensors beside those strictly necessary for control purposes. Using geometric nonlinear techniques, a set of diagnostic signals has been designed, from which a suitable subset of residuals has been selected, satisfying the desired FDI architecture and functionality.
The performance of the designed FDI system has been demonstrated by numerical simulations performed on the identified model of the real plant, including actual levels of input and measurement noise.

\section{ACKNOWLEDGEMENTS}

This work was supported by the EU project EUIST-2001-32122 IFATIS.

\section{REFERENCES}

Bonivento, C., M. Capiluppi, L. Marconi and A. Paoli (2004): An integrated design approach to multilevel Fault Tolerant Control of distributed systems. 16 $6^{\text {th }}$ IFAC World Congress. Praha, CZ.

De Persis, C. and A. Isidori (2001): A geometric approach to nonlinear fault detection and isolation. IEEE Trans. on Automatic Control. 46(6), 853-865.

Hamelin, F., H. Jamouli and D. Sauter (2004): The two tanks pilot plant. IFATIS report IFAN014R01.

Mattone, R. and A. De Luca (2003): Detection and isolation of sensor faults in nonlinear systems: A case study. Workshop on Advanced Control and Diagnosis. Duisburg, D. pp. 49-54.

Mattone, R. and A. De Luca (2004): Fault set detection and isolation for nonlinear systems. Automatica. Submitted for publication. 